\title{
Optimized Criteria for Sentinel Lymph Node Biopsy in Patients with Clinically Node Negative Breast Cancer
}

\author{
Jeong Suk Kim, M.D., Moo Hyun Lee, M.D., Sun Hee Kang, M.D., Ph.D., Jihyoung Cho, M.D. \\ Department of Surgery, Keimyung University School of Medicine, Daegu, Korea
}

Purpose: Sentinel lymph node biopsy (SLNB) is a well-established staging procedure for patients with early breast cancer who have clinically negative axillary lymph node. However, no consensus exists about the number of sentinel lymph nodes (SLN) that should be removed based on radioactivity counts in breast cancer. We reviewed and analyzed cases in which more than one SLN was detected and there was at least one pathologically positive node. Methods: We retrospectively studied breast cancer patients who underwent lymphoscintigraphy with injection of a radioactive colloid and SLNB along with intraoperative determination of radioactive counts of lymph nodes using a gamma probe between 2006 and 2018. In total 326 patients with more than one radioactive SLN were enrolled in this study. Results: Fifty-four patients had nodal metastases, of whom 46 (85.2\%) had metastases in the hottest lymph node. All metastatic SLNs were identified as one of the first three lymph nodes dissected. The lowest radioactive count of a positive SLN corresponded to $10 \%$ of that of the hottest node. Conclusion: We suggest that removal of the first three lymph nodes or nodes covered by the "10\% rule" is sufficient in SLNB for patients with breast cancer.

Key Words: Breast neoplasms, Lymph node excision, Sentinel lymph node biopsy

\section{INTRODUCTION}

Breast cancer prognosis is mainly determined by axillary lymph node status [1]. The sentinel lymph node (SLN) has been defined as the first lymph node to receive lymphatic drainage from a tumor bed. Commonly, there can be more than one SLN, and therefore, it is currently defined as any lymph node or first set of nodes that receives direct lymphatic drainage from a primary tumor [2].

Sentinel lymph node biopsy (SLNB) is a well-established procedure for the staging of the axilla in early stage breast cancer and has replaced axillary lymph node dissection (ALND) as the standard of care in patients with clinically negative axillary lymph node [3]. SLNB limits the extent of axillary surgery compared with complete ALND, thus reducing the associated morbidity [4]. Following the American College of Surgeons Oncology Group (ACOSOG) Z0011 trial, the role of ALND after SLNB has been further reduced $[5,6]$. However, because

Correspondence: Jihyoung Cho, M.D.

Department of Surgery, Keimyung University School of Medicine, 56 Dalseongro, Jung-gu, Daegu 41931, Korea

Tel: +82-53-258-4708, Fax: +82-53-258-4710, E-mail: chojh0404@dsmc.or.kr

This work was supported by a National Research Foundation of Korea (NRF) grant funded by the Korea Government (MSIP) (no. 2018R1C1B5086617).

Received: Oct 16, 2020 Revised: Nov 5, 2020 Accepted: Jan 24, 2021 no consensus exists about the number of SLNs that should be removed based on the relative radioactivity counts registered during surgery, the " $10 \%$ of the hottest nodes rule" is often used $[7,8]$. To determine the frequency at which radioactivity counts can actually be used to detect malignant SLNs and to define the optimal criteria for removal of radioactive lymph nodes, we reviewed and analyzed the frequency and distribution of radioactive malignant SLNs in patients with breast cancer who had more than one radioactive SLN and at least one pathologically positive lymph node.

\section{METHODS}

We retrospectively reviewed the medical records of 1,687 patients with breast cancer who underwent lymphoscintigraphy with injection of a radioactive colloid and SLNB with intraoperative determination of the radioactive count of lymph nodes using a gamma probe (Neoprobe, Mammotome, Cincinnati, USA) at Keimyung University Dongsan Hospital between January 2006 and December 2018. The study was approved by our Institutional Review Board (IRB No. 202010-001-002).

SLNB was performed in all patients with early-stage breast cancer without clinically positive axillary lymph nodes or advanced disease. 
Patients with no detectable SLNs or only one SLN were excluded. A total of 326 patients who had more than one radioactive SLN were enrolled in this study. SLN-related parameters such as the number, radioactivity, order, and pathological status of SLNs were prospectively recorded. An incision was made over the hottest area identified by the gamma probe, and SLNs were retrieved for intraoperative frozen section evaluation. Patients who had positive SLNs underwent ALND. We analyzed the clinical and pathological data of patients, including data on age, tumor-node-metastasis stage, hormone receptor and human epidermal growth factor receptor type 2 (HER2) status, histologic type, number of SLNs, relative radioactivity counts, and presence of metastasis in SLNs.

\section{RESULTS}

The clinicopathological characteristics of the 326 patients with more than one radioactive SLN are described in Table 1. The mean age of the patients was 53.0 (standard deviation, \pm 11.1 ) years. The T

Table 1. Clinicopathological characteristics of patients with more than one radioactive sentinel lymph node $(n=326)$

\begin{tabular}{lc}
\hline Characteristic & No. of patients (\%) \\
\hline Mean age \pm SD (yr) & $53.0 \pm 11.1$ \\
Tumor stage & \\
Tis & $13(4.0)$ \\
T1 & $191(58.6)$ \\
T2 & $117(35.9)$ \\
T3 & $4(1.2)$ \\
T4 & $1(0.3)$ \\
Histology & \\
Invasive ductal carcinoma & $287(88.0)$ \\
Invasive lobular carcinoma & $23(7.1)$ \\
Others & $16(4.9)$ \\
Nodal stage & \\
N0 & $272(83.4)$ \\
N1mic or N1 & $49(15.0)$ \\
N2 & $4(1.2)$ \\
N3 & $1(0.3)$ \\
Hormone receptor status & \\
Negative & $84(25.7)$ \\
Positive & $241(74.0)$ \\
Unknown & $1(0.3)$ \\
HER2 status & $183(56.1)$ \\
Negative & $10(3.1)$ \\
Equivocal & $133(40.8)$ \\
Positive &
\end{tabular}

$\mathrm{SD}=$ standard deviation; HER2 = human epidermal growth factor- 2 . stage of the primary tumor was Tis in 13 patients (4.0\%), T1 in $191 \mathrm{pa-}$ tients (58.6\%), T2 in 117 patients (35.9\%), and T3 or T4 in 5 patients (1.5\%). Invasive ductal carcinoma was the most common histological tumor type (287 of 326), and invasive lobular carcinoma was diagnosed in 23 patients. The N stage was N0 in 272 (83.4\%) patients, N1mic or N1 in 49 patients (15.0\%), N2 in 4 patients (1.2\%), and N3 in 1 patient (0.3\%). Hormone receptor status was negative in 84 patients (25.7\%) and positive in 241 patients (74.0\%). HER2 status was negative in 183 patients (56.1\%), equivocal in 10 patients (3.1\%), and positive in 133 patients (40.8\%).

Among 326 patients with more than one radioactive SLN, 54 (16.6\%) had nodal metastases. Among patients with metastatic SLNs, the hottest SLN showed metastasis in 85.2\% (46 of 54) of cases, whereas $14.8 \%$ (8 of 54) of cases involved metastasis in SLNs other than the hottest SLN (Figure 1). Six of 256 patients (2.0\%) with two radioactive SLNs showed metastasis in the non-hottest SLN and no metastasis in

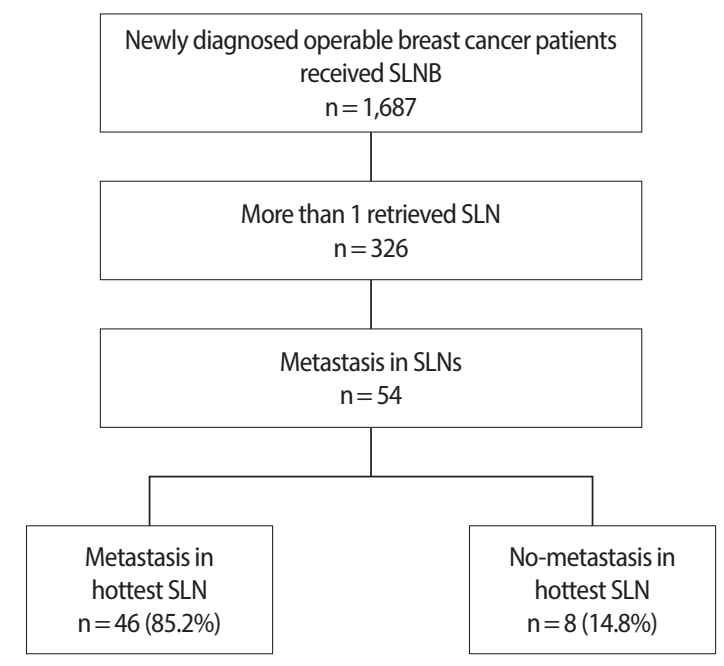

Figure 1. Study profile. SLNB = sentinel lymph node biopsy; SLN= sentinel lymph node.

Table 2. Distribution of malignancy in radioactive SLNs of patients with more than one radioactive SLNs $(n=326)$

\begin{tabular}{lcc}
\hline No. of radioactive SLNs & No. of patients & $\begin{array}{c}\text { No. of patients with positive } \\
\text { non-hottest SLN and negative } \\
\text { hottest SLN (\%) }\end{array}$ \\
\hline 2 & 256 & $6(2.0)$ \\
3 & 55 & $2(4.0)$ \\
4 & 13 & 0 \\
$\geq 5$ & 2 & 0 \\
Total & 326 & $8(2.5)$ \\
\hline
\end{tabular}

$\mathrm{SLN}=$ sentinel lymph node. 
Table 3. Cases with non-metastatic hottest SLN and metastatic non-hottest SLN

\begin{tabular}{|c|c|c|c|c|c|c|c|c|c|}
\hline \multirow{2}{*}{ Case number } & \multirow{2}{*}{ Histology } & \multirow{2}{*}{$\begin{array}{c}\text { Hormone } \\
\text { receptor } \\
\text { status }\end{array}$} & \multirow{2}{*}{ HER2 status } & \multirow{2}{*}{ Staging } & \multirow{2}{*}{$\begin{array}{l}\text { Activity of the } \\
\text { hottest SLN }\end{array}$} & \multicolumn{3}{|c|}{ Relative activity of the retrieved SLNs (\%) } & \multirow{2}{*}{$\begin{array}{l}\text { Total number } \\
\text { of metastatic } \\
\text { lymph nodes }\end{array}$} \\
\hline & & & & & & First & Second & Third & \\
\hline 1 & IDC & Positive & Positive & T2N1M0 & 2,600 & 100 & $69^{*}$ & & 2 \\
\hline 2 & IDC & Positive & Positive & T1N1M0 & 780 & 100 & $32^{*}$ & & 0 \\
\hline 3 & IDC & Positive & Positive & T2N1M0 & 6,000 & 100 & 65 & $23^{*}$ & 0 \\
\hline 4 & IDC & Positive & Negative & T2N3M0 & 1,600 & 100 & $94^{*}$ & & 14 \\
\hline 5 & IDC & Positive & Negative & T1N1M0 & 10,000 & 100 & 60 & $55^{*}$ & 0 \\
\hline 6 & IDC & Positive & Negative & T2N1M0 & 19,000 & 100 & $23^{*}$ & & 2 \\
\hline 7 & IDC & Positive & Negative & T1N1M0 & 4,000 & 100 & $10^{*}$ & & 1 \\
\hline 8 & Metaplastic & Negative & Negative & T1N1M0 & 12,000 & 100 & $42^{*}$ & & 1 \\
\hline
\end{tabular}

$\mathrm{SLN}=$ sentinel lymph node; HER2 = human epidermal growth factor-2; IDC = invasive ductal carcinoma.

*Metastatic lymph node.

the hottest SLN. Of the 55 patients with three radioactive SLNs, two (4.0\%) showed metastasis in non-hottest SLNs but showed no metastasis in the hottest SLN. Fifteen patients had more than three radioactive SLNs, and they showed no metastasis in the non-hottest SLN (Table 2).

The cases in which the non-hottest SLNs were metastatic and the hottest SLNs were non-metastatic are shown in Table 3. Among the eight patients with no metastasis in the hottest SLN, six showed metastasis in the second hottest SLN and two showed metastasis in the third-hottest SLN. The lowest percentage of radioactivity in a metastatic SLN was $10 \%$, corresponding to the second hottest SLN of one of the patients.

\section{DISCUSSION}

ALND is associated with the development of lymphedema in patients with early-stage breast cancer [9]. After the ACOSOG Z0011 study, the importance of ALND after SLNB has been minimized, and several studies have shown that the number of patients undergoing ALND for pathologically positive SLNs has significantly decreased $[5,6,10]$. Studies that investigated the omission of SLNB for selected patients with breast cancer have also been published [11,12]. Reducing the number of dissected SLNs during SLNB is known to decrease morbidity.

SLNB has traditionally been the standard axillary staging procedure for patients with clinically node-negative breast cancer. Fortunately, the ability to identify SLNs has improved owing to increased surgical experience and refinements in nuclear imaging techniques, including injection methods and injection volume. Although the concept is simple, there is a large variability in how the procedure is performed at different institutions and in the radioactive threshold used for SLN removal. The most popular guideline is the " $10 \%$ of the hottest nodes rule," which is used to minimize the chances of morbidity related to ALND [7]. In our study, the most metastatic SLNs had the highest radioactivity in $85.2 \%$ of cases, and the hottest lymph node was not pathologically positive in $14.8 \%$ of cases. The lowest relative radioactivity in a metastatic non-hottest SLN was $10 \%$.

Regardless of the radioactive status of the SLN, the following question remains to be answered: How many sentinel nodes should be removed to achieve appropriate accuracy of axillary staging without negating the potential benefits of SLNB? Previously published studies have shown that $97 \%-98 \%$ of positive SLNs are discovered in one of the first three nodes examined and that it is rare to find a positive SLN after the first three nodes are identified as negative [2,4,13-15]. Ban et al. [2] suggested that the total number of SLNs excised should be limited to no more than four. In our study, all positive SLNs were identified as one of the first three lymph nodes dissected from patients with node-positive disease, consistent with previously reported findings.

Our study showed that there were only a few cases in which the hottest lymph node was not pathologically positive. In addition, all metastatic SLNs were identified as one of the first three lymph nodes dissected from patients with node-positive disease. To remove only the nodes that are most likely to be metastatic and to reduce the morbidity of axillary dissection, we suggest that removal of the first three lymph nodes or nodes covered by the " $10 \%$ rule" is sufficient for SLNB in patients with breast cancer. 


\section{CONFLICT OF INTEREST}

The authors declare that they have no competing interests.

\section{REFERENCES}

1. Collins CD. The sentinel node in breast cancer: an update. Cancer Imaging 2005;5:S3-9.

2. Ban EJ, Lee JS, Koo JS, Park S, Kim SI, Park BW. How many sentinel lymph nodes are enough for accurate axillary staging in t1-2 breast cancer? J Breast Cancer 2011;14:296-300.

3. Colakoglu MK, Guven E, Akgul GG, Dogan L, Gulcelik MA. Biological subtypes of breast cancer and sentinel lymph node biopsy. Eur J Breast Health 2018;14:100-4.

4. Yi M, Meric-Bernstam F, Ross MI, Akins JS, Hwang RF, Lucci A, et al. How many sentinel lymph nodes are enough during sentinel lymph node dissection for breast cancer? Cancer 2008;113:30-7.

5. Giuliano AE, Ballman KV, McCall L, Beitsch PD, Brennan MB, Kelemen PR, et al. Effect of axillary dissection vs no axillary dissection on 10-year overall survival among women with invasive breast cancer and sentinel node metastasis: the ACOSOG Z0011 (alliance) randomized clinical trial. JAMA 2017;318:918-26.

6. Joyce DP, Lowery AJ, McGrath-Soo LB, Downey E, Kelly L, O'Donoghue GT, et al. Management of the axilla: has Z0011 had an impact? Ir J Med Sci 2016;185:145-9.

7. McMasters KM, Tuttle TM, Carlson DJ, Brown CM, Noyes RD, Glaser RL, et al. Sentinel lymph node biopsy for breast cancer: a suitable alternative to routine axillary dissection in multi-institu- tional practice when optimal technique is used. J Clin Oncol 2000; 18:2560-6

8. Dutta R, Kluftinger A, MacLeod M, Kindrachuk G, Baliski C. Revisiting the " $10 \%$ rule" in breast cancer sentinel lymph node biopsy: an approach to minimize the number of sentinel lymph nodes removed. Am J Surg 2012;203:623-7.

9. Göker M, Devoogdt N, Van de Putte G, Schobbens JC, Vlasselaer J, Van den Broecke R, et al. Systematic review of breast cancer related lymphoedema: making a balanced decision to perform an axillary clearance. Facts Views Vis Obgyn 2013;5:106-15.

10. Subhedar P, Stempel M, Eaton A, Morrow M, Gemignani ML. Do the ACOSOG Z0011 criteria affect the number of sentinel lymph nodes removed? Ann Surg Oncol 2015;22:470-5.

11. Nakagawa M, Morimoto M, Takechi H, Tadokoro Y, Tangoku A. Preoperative diagnosis of sentinel lymph node (SLN) metastasis using 3D CT lymphography (CTLG). Breast Cancer 2016;23:519-24.

12. Nakauchi C, Naoi Y, Shimazu K, Tsunashima R, Nishio M, Maruyama N, et al. Development of a prediction model for lymph node metastasis in luminal A subtype breast cancer: the possibility to omit sentinel lymph node biopsy. Cancer Lett 2014;353:52-8.

13. Dabbs DJ, Johnson R. The optimal number of sentinel lymph nodes for focused pathologic examination. Breast J 2004;10:186-9.

14. McCarter MD, Yeung H, Fey J, Borgen PI, Cody HS 3rd. The breast cancer patient with multiple sentinel nodes: when to stop? J Am Coll Surg 2001;192:692-7.

15. Woznick A, Franco M, Bendick P, Benitez PR. Sentinel lymph node dissection for breast cancer: how many nodes are enough and which technique is optimal? Am J Surg 2006;191:330-3. 